\title{
Malignant Childhood Hemangiopericytoma
}

National Cancer Institute

\section{Source}

National Cancer Institute. Malignant Childhood Hemangiopericytoma. NCI Thesaurus.

Code C8090.

A malignant hemangiopericytoma occurring in childhood. 